\section{Tox-Zentrum 2000}

\section{Zum Jahresbericht des Schweizerischen Toxikologischen Informationszentrums}

C. Rauber, M. Guirguis, S. Schnorf, J. P. Lorent, P. J. Meier-Abt, H. Kupferschmidt

\section{Zusammenfassung}

Im Jahr 2000 beantwortete das Tox-Zentrum 30935 Anfragen zu Vergiftungen. 3926 Vergiftungsfälle konnten aufgrund der Rückmeldungen von Ärztinnen und Ärzten bezüglich Symptome und Schweregrad der Vergiftungsverläufe analysiert und in die anonymisierte Vergiftungsdatenbank aufgenommen werden. Bei Kindern verliefen 26 Vergiftungen schwer und ein Fall tödlich. Bei Erwachsenen wurden 242 schwere und 15 tödliche Vergiftungsverläufe registriert. Die häufigsten Vergiftungsursachen waren Medikamente, Haushaltprodukte (v.a. Kinder), technische und gewerbliche Produkte sowie Genussmittel, Drogen und Alkohol (v.a. Erwachsene). Genauer analysiert wurden vor allem Methadonvergiftungen bei Kleinkindern, Vergiftungen mit selektiven Serotoninwiederaufnahmehemmern und Fehler bei der Anwendung von Medikamenten.

Der vollständige Jahresbericht findet sich auf unserer Website (www.toxi.ch).

\section{Einleitung}

Im Jahr 2000 erhielt das Tox-Zentrum 30935 Anfragen zu potenziellen und tatsächlichen Vergiftungen. 24756 Anfragen betrafen Fälle (23 667 Menschen, 1089 Tiere) mit stattgehabtem Giftkontakt. 5985 Fälle verliefen harmlos. Bei 18771 Patienten ging die Giftexposition mit einer potenziellen Gesundheitsgefährdung einher. Insgesamt 9243 Anfragen kamen von Ärztinnen und Ärzten, darunter 7101 Anfragen aus Spitälern und 2142 aus der Praxis. Bei 6524 ärztlichen Anfragen lag eine potentielle oder manifeste Vergiftung vor. In diesen Fällen erhielten die behandelnden Ärztinnen und Ärzte eine schriftliche Bestätigung der telefonischen Beratung, zusammen mit dem Wunsch nach einem Verlaufsbericht. In 68\% dieser Fälle liessen die Ärztinnen und Ärzte dem Tox eine Rückmeldung über den Verlauf der Vergiftung

Korrespondenz:

Dr. H. Kupferschmidt

Schweizerisches Toxikologisches Informationszentrum

Freiestrasse 16

Postfach

CH-8028 Zürich

E-mail: info@toxi.ch zukommen. Jeder dieser klinischen Berichte wurde nach Kausalität und Schweregrad beurteilt. 3926 symptomlose und symptomatische human-medizinische Fälle mit gesicherter und wahrscheinlicher Kausalität wurden bezüglich des klinischen Verlaufs weiter analysiert.

\section{Noxen und Schweregrad der Vergiftungsverläufe}

In Tabelle 1 sind die Schweregrade der Vergiftungsverläufe nach Einnahme verschiedener Noxen zusammengestellt. 1027 Vergiftungsfälle betrafen Kinder ( $\leq 16$ Jahre). 2899 Vergiftungen ereigneten sich bei Erwachsenen.

Bei den Kindern kam es meistens zu einem symptomlosen (44,5\%) oder leichten $(45,1 \%)$ Vergiftungsverlauf. Mittelschwere Intoxikationen ereigneten sich in $80(7,8 \%)$ und schwere Intoxikationen in $26(2,5 \%)$ Fällen. Ein Kleinkind starb nach akzidenteller Einnahme einer Überdosis von Methadon (s. unten).

Je nach Altersklasse der Kinder waren die involvierten Noxen sehr unterschiedlich. Bei Kindern unter 12 Jahren (823 Fälle) beobachteten wir Unfälle mit sämtlichen Noxen aus der häuslichen Umgebung. Die Palette reichte von relativ wenig giftigen Produkten wie kleine Dosen harmloser Medikamente, Kosmetika und Zimmerpflanzen bis hin zu gefährlichen Giften wie stark ätzenden Produkten oder stark toxischen Medikamenten. Bei den Haushaltsprodukten muss ein besonderes Augenmerk auf die neuen antibakteriellen Reinigungsmittel gerichtet werden. Erste Erfahrungen mit diesen Produkten deuten darauf hin, dass die desinfizierenden Zusätze zu verstärkten Augenund Schleimhautreizungen führen können.

Bei den 204 Jugendlichen (12-16 Jahre) dominierten Abusus und Suizidversuche als hauptsächliche Vergiftungsursachen und dementsprechend zwei ganz unterschiedliche Noxengruppen. Beim Missbrauch verursachten Alkohol, Dextromethorphan, halluzinogene Pilze, Morphin, Kokain und Methylphenidat (Ritalin ${ }^{\circledR}$ ) schwere und mittelschwere Intoxikationen. Erstmals registrierte das Tox zudem Anfragen zu A2 (Benzylpiperazin), 2C-B (Bromo-Dimethoxyphenethylamin) und zur Thaipille (Methamphetamin). Bei den Noxen, die in suizidaler Absicht eingenommen wurden, war das Spektrum breit. Die Jugendlichen nahmen ein, was in der Hausapotheke verfügbar war. So registrierten wir hier in absteigender Häufigkeit schwere und mittelschwere Fälle mit Mefenaminsäure, Paracetamol, Benzodiazepinen, Betablockern, selektiven Serotoninwiederaufnahmehemmern und trizyklischen Antidepressiva.

Bei den Erwachsenen verliefen lediglich 350 $(12,1 \%)$ Vergiftungen ohne Symptome. Leichte Vergiftungsverläufe traten in 1642 (56,6\%), mittelschwere in $650(22,4 \%)$, schwere in $242(8,4 \%)$, und tödliche in 15 (0,5\%) Fällen auf. 9 Todesfälle waren auf die beabsichtigte Einnahme von Medikamenten zurückzuführen. In je einem Fall führten Suchtmittel, der Cholinesterasehemmer Parathion sowie kon- 


\section{Abbildung 1}

Verlauf der Monointoxikationen mit SSRI ( $\mathrm{n}=197$ ).

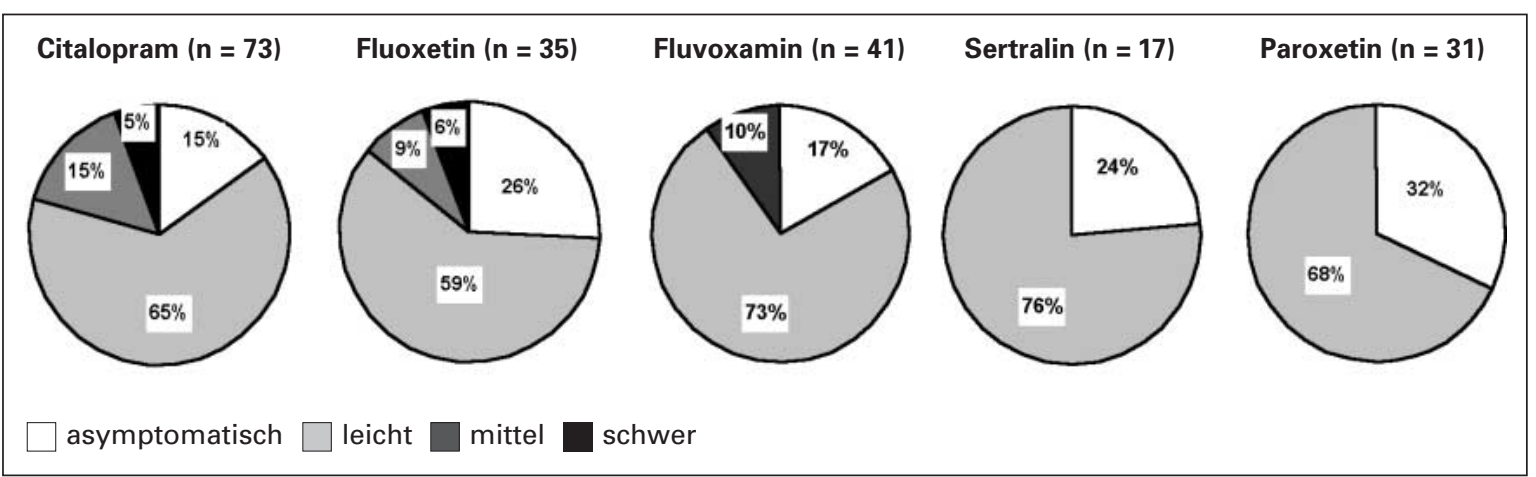

zentrierte Ameisensäure zum Tod. Weitere Todesfälle ereigneten sich infolge einer akzidentellen Methotrexatüberdosierung, einem Naproxen-induzierten Leberversagen und einem Arbeitsunfall mit Flusssäure.

\section{Spezielle Vergiftungsprobleme im Jahre 2000}

Methadonvergiftungen beim Kleinkind

Wie bereits erwähnt ereignete sich im Berichtsjahr ein tödlicher Kinderunfall mit Methadon. Wir nahmen diesen tragischen Fall zum Anlass, die am Tox registrierten Kleinkinderunfälle mit Methadon während der letzten 10 Jahre zusammenzustellen (Tab. 2). Es fanden sich insgesamt 34 Methadonfälle mit ärztlich gut dokumentiertem Intoxikationsverlauf. Die Kleinkinder konsumierten das Methadon in der Regel aus unvorsichtig aufbewahrten Fläschchen mit Methadonpräparaten, die den Eltern im Rahmen ihrer Opiatentzugsbehandlung von Ärzten und/oder Apothekern nach Hause mitgegeben wurden. Bei 15 Kleinkindern verlief die Methadonintoxikation mit mittelschweren, schweren oder sogar tödlichen Symptomen. Die häufigsten Symptome waren Somnolenz bis Koma, Miosis und Ateminsuffizienz. Daneben traten auch Erbrechen, Harnretention, Kreislaufversagen und als Komplikation je einmal eine Aspirationspneumonie und eine irreversible hypoxische Hirnschädigung auf. 2 Kleinkinder verstarben an den akzidentellen Methadonüberdosierungen.

Methadoningestionen sind beim Kleinkind zwar ein seltenes Ereignis, stellen aber, wie unsere Fälle zeigen, ein ernst zu nehmendes Gefahrenpotential dar. Methadon kann bei Kleinkindern bereits in einer Dosierung von $10 \mathrm{mg}$ zu einer tödlichen Vergiftung führen. Die betroffenen Kinder trinken meistens die ihren Eltern abgegebene, trinkfertige und oft mit Sirup gesüsste Methadonlösung. Die Methadonsubstitution als eine etablierte und wichtige Therapie soll hier in keiner Art und Weise in Frage gestellt werden. Ärzte und Apotheker, die an Eltern Methadon nach Hause abgeben, sollten aber alle möglichen Vorsichtsmassnahmen treffen, um Kleinkinder vor einer akzidentellen Methadoneinnahme zu schützen. Das heisst, wenn immer möglich sollte bei Eltern von Kleinkindern auf die Methadonabgabe nach Hause verzichtet werden. Ist dies nicht möglich, so sollten die Eltern bei der Abgabe explizit auf die Gefahr einer akzidentellen Einnahme durch ihr Kind aufmerksam gemacht und das Methadon nur in Fläschchen mit kindersicherem Verschluss abgegeben werden.

Selektive Serotoninwiederaufnahmehemmer In der Vergiftungsstatistik 2000 sind 333 Intoxikationen bei Erwachsenen mit einem Antidepressivum als toxikologisch wichtigster Noxe ausgewiesen. Ausser den klassischen trizyklischen Antidepressiva, deren quantitative und qualitative Toxizität gut bekannt ist, kam es bei zunehmender therapeutischer Verwendung auch vermehrt zu Intoxikationen mit selektiven Serotoninwiederaufnahmehemmern (SSRI).

Zur Verbesserung der Risikoabschätzung analysierten wir deshalb 197 gut dokumentierte SSRIMonointoxikationen aus der Kasuistik des Tox von 1984 bis 2000. Es traten 18 mittelschwere und 6 schwere Intoxikationen auf, in denen vorwiegend Krampfanfälle und EKG-Störungen $\mathrm{zu}$ beobachten waren. Bei den Erwachsenen traten diese Symptome bei Einnahmedosen über $280 \mathrm{mg}$ Citalopram, $60 \mathrm{mg}$ Fluoxetin und 1,5 g Fluvoxamin auf. Sertralin und Paroxetin führten nicht zu Krämpfen oder EKGStörungen, sondern verursachten nur leichte Intoxikationen. Abbildung 1 zeigt die unterschiedliche Häufigkeit, mit der diese fünf SSRI zu einem mittelschweren oder schweren Verlauf führten, nämlich in 20\% bei Citalopram, 15\% bei Fluoxetin und 10\% bei Fluvoxamin, jedoch nie bei Sertralin und Paroxetin.

Fehler bei der Anwendung von Medikamenten Das Tox-Zentrum erhält regelmässig Anfragen von Fachpersonen und Laien, wenn bei der Anwendung von Medikamenten Fehler und Missgeschicke passieren. In einer Pilotstudie wurden diese Anfragen aus drei Jahren (1998-2000) untersucht. Es wurden nur Fälle in die Analyse eingeschlossen, bei denen der Anfrager das Ereignis als Fehler deklarierte. Die Gesamtzahl der Fälle betrug 1035. Es handelte sich am häufigsten um eine irrtümliche Überdosierung oder eine Verwechslung (des Medikamentes oder des Pa- 
Tabelle 1

Vergleich Noxengruppen/Vergiftungsschweregrad bei Erwachsenen und Kindern.

\begin{tabular}{lrrrrrrrrrrrr}
\hline & \multicolumn{1}{c}{ Erwachsene } & \multicolumn{1}{c}{ Kinder } & & \multicolumn{3}{c}{ Total } \\
\hline Schweregrad & 0 & L & M & S & T & 0 & L & M & S & T & \\
\hline Medikamente & 264 & 1126 & 424 & 161 & 11 & 263 & 263 & 58 & 15 & 1 & 2586 & $(65,9 \%)$ \\
\hline Haushaltprodukte & 28 & 123 & 20 & 4 & - & 83 & 89 & 6 & 6 & - & 359 & $(9,1 \%)$ \\
\hline Technische und gewerbliche Produkte & 20 & 169 & 46 & 10 & 2 & 24 & 26 & 3 & 1 & - & 301 & $(7,7 \%)$ \\
\hline Genussmittel, Drogen und Alkohol & 9 & 72 & 83 & 50 & 1 & 16 & 19 & 4 & 2 & - & 256 & $(6,6 \%)$ \\
\hline Pflanzen & 8 & 27 & 17 & - & - & 25 & 15 & 3 & - & - & 95 & $(2,4 \%)$ \\
\hline Pilze & 3 & 17 & 26 & 4 & - & 4 & 1 & 3 & 2 & - & 60 & $(1,5 \%)$ \\
\hline Landwirtschaft und Gartenbau & 6 & 19 & 9 & 6 & 1 & 21 & 6 & - & - & - & 68 & $(1,7 \%)$ \\
\hline Körperpflegemittel und Kosmetika & 2 & 19 & 3 & - & - & 17 & 10 & - & - & - & 51 & $(1,3 \%)$ \\
\hline Gift-)Tiere & 1 & 15 & 4 & 4 & - & 2 & 7 & 3 & - & - & 36 & $(0,9 \%)$ \\
\hline Nahrungsmittel und Getränke & 2 & 4 & 3 & 1 & - & 1 & 6 & - & - & - & 17 & $(0,4 \%)$ \\
\hline Veterinärarzneimittel & 3 & 1 & 4 & - & - & - & - & - & - & - & 8 & $(0,2 \%)$ \\
\hline Andere oder unbekannte Noxen & 4 & 50 & 11 & 2 & - & 1 & 21 & - & - & - & 89 & $(2,3 \%)$ \\
\hline Total & 350 & 1642 & 650 & 242 & 15 & 457 & 463 & 80 & 26 & 1 & 3926 & $(100 \%)$ \\
\hline
\end{tabular}

Schweregrad des Verlaufs: 0 = asymptomatisch, $\mathrm{L}=$ leicht, $\mathrm{M}=$ mittel, $\mathrm{S}=$ schwer, $\mathrm{T}$ = tödlich

\section{Tabelle 2}

Verlauf bei Kleinkinderunfällen mit Methadon 1991-2000 ( $\mathrm{n}=34)$.

\begin{tabular}{|c|c|c|c|}
\hline Verlauf & $\begin{array}{l}\text { Anzahl } \\
\text { Fälle }\end{array}$ & Leitsymptome & Bemerkungen \\
\hline Asymptomatisch & 6 & - & $\begin{array}{l}5 \text { der } 6 \text { Kinder wurden frühzeitig } \\
\text { dekontaminiert, eines erhielt prophylaktisch } \\
\text { Narcan }^{\circledR}\end{array}$ \\
\hline Leicht & 13 & Somnolenz, Erbrechen, Miosis, Gangstörungen & $\begin{array}{l}7 \text { der } 13 \text { Kinder wurden frühzeitig dekontaminiert. } \\
6 \text { Kinder erhielten vorsichtshalber Narcan }{ }^{\circledR}\end{array}$ \\
\hline Mittel & 4 & Koma (GCS 8-9), mittelgradige Atemdepression & 3 Kinder erhielten Narcan $^{\circledR}$ \\
\hline Schwer & 9 & $\begin{array}{l}\text { Koma (GCS } \leq 7 \text { ), Ateminsuffizienz, Lungenödem, } \\
\text { hypoxische Hirnschädigung }\end{array}$ & $\begin{array}{l}8 \text { Kinder erhielten } \operatorname{Narcan}^{\circledR} \text {, } \\
2 \text { wurden intubiert und beatmet }\end{array}$ \\
\hline Tödlich & 2 & Atem-, Kreislaufversagen & $\begin{array}{l}\text { Beide Kinder hatten vor dem Eintreffen } \\
\text { des Arztes ein Atem- und Kreislaufversagen }\end{array}$ \\
\hline
\end{tabular}

tienten), meistens mit Psychopharmaka oder Analgetika. Urheber des Irrtums war in 68\% der Fälle ein medizinischer Laie, in den übrigen Fällen eine Fachperson (Arzt, Apotheker, Pflegeberuf). In rund einem Drittel der Fälle handelte es sich um einen Fehler im Rahmen der Selbstapplikation, bei zwei Dritteln waren die Opfer des Irrtums Drittpersonen. 46\% der betroffenen Patienten waren Kinder ( $\leq 16$ Jahre). Anrufe wegen Fehlern bei der Anwendung von Medikamenten waren zwischen 19.00 und 23.00 Uhr doppelt so häufig wie während des Tages. Eine Erklärung dafür könnte sein, dass nach Feierabend andere Ratgeber nicht mehr zugänglich sind, während das Tox-Zentrum rund um die Uhr zur Verfügung steht. Die Daten zeigen, dass das Tox-Zentrum nicht nur bei Vergiftungen, sondern auch bei fehlerhafter Medikation eine wichtige Rolle in der Notfallberatung spielt.

\section{Schlussfolgerungen}

Humantoxikologische Daten zu Überdosierungen oder Fehlapplikationen können aus naheliegenden Gründen nicht aus experimentellen Studien erhoben werden. Im Rahmen von Spontanmeldungen kann das Tox-Zentrum auch keine zuverlässigen Angaben zur absoluten Häufigkeit von Vergiftungen und Fehlapplikationen von Medikamenten machen. Die Anfragen und Meldungen können aber als Signale für 
aktuelle und neu auftretende Vergiftungsprobleme in der Bevölkerung und damit als Frühwarnsystem in der Toxikovigilanz dienen. Dies trifft insbesondere für den Missbrauch neuer Drogen, die Verträglichkeit neuer Medikamente und die Bedenklichkeit neuer Produkte in Haushalt, Gewerbe, Industrie und Landwirtschaft zu. Die Methadonvergiftungen bei Kleinkindern zeigen einmal mehr, dass das Spontanmeldesystem auch bei etablierten Wirkstoffen wichtige Hinweise auf aktuelle und potentiell-vermeidbare Vergiftungssituationen liefert. Wertvoll sind schliesslich alle ärztlichen Rückmeldungen über die Vergif- tungsverläufe. Sie erlauben dem Tox-Zentrum den kontinuierlichen Weiterausbau der Vergiftungsdatenbank als Basis für die humantoxikologische Beurteilung von alten und neuen chemischen Substanzen und Wirkstoffen und die vergleichende Toxizitätsanalyse von einzelnen Wirkstoffen einer Substanzklasse wie zum Beispiel der Serotoninwiederaufnahmehemmer. Das Tox-Zentrum dankt allen Ärztinnen und Ärzten sowie Apothekerinnen und Apothekern in Spital und Praxis für ihre Mitarbeit zur kontinuierlichen Verbesserung der Arzneimittel- und Chemiesicherheit in der Schweiz.

\section{Rapport du Tox}

\section{A propos du rapport annuel 2000 du Centre suisse d'information toxicologique \\ C. Rauber, M. Guirguis, S. Schnorf, J. P. Lorent, P. J. Meier-Abt, H. Kupferschmidt}

\section{Introduction}

En 2000, le Tox a reçu 30935 appels au sujet d'intoxications manifestes ou potentielles. 24756 demandes d'information concernaient des expositions effectives (23 667 personnes, 1089 animaux). 5985 cas étaient anodins. Chez 18771 patients, un risque pour la santé était présent.

9243 appels au total provenaient des médecins (hospitaliers dans 7101 et praticiens dans 2142 cas). Une intoxication potentielle ou manifeste était la raison de l'appel du médecin dans 6524 cas. La confirmation écrite de la réponse, dans ces cas, était accompagnée du souhait de recevoir en retour des informations sur l'évolution du cas. Le taux de réponse a été de $68 \%$. Chacune des observations médicales reçues a été classée selon sa causalité et son degré de gravité. 3926 observations chez l'homme à causalité vraisemblable ou certaine ont ainsi pu être étudiées.

\section{Agents responsables et gravité des intoxications}

Le tableau 1 montre la gravité des intoxications selon le type d'agents responsables. 1027 cas concernaient les enfants ( $\leq 16$ ans). 2899 intoxications ont été observées chez l'adulte.

L'évolution chez les enfants était en général bénigne (sans symptômes $44,5 \%$, intoxication légère $45,1 \%$ ). Des intoxications de gravité moyenne ont été vues dans 80 cas $(7,8 \%)$ et des intoxications graves 26 fois (2,5\%). Un jeune enfant est mort à la suite de l'ingestion accidentelle de méthadone (voir plus bas).

Les produits en cause varient fortement selon l'âge des enfants. Chez les petits en-dessous de douze ans (823 cas), on constate des accidents avec tous les produits de l'environnement domestique. Une large palette comprend aussi bien des produits assez peu dangereux aux doses généralement absorbées, comme les médicaments faibles, les produits cosmétiques ou les plantes d'appartement, que des poisons
Correspondance:

Dr H. Kupferschmidt

Centre suisse d'information toxicologique

Freiestrasse 16

Case postale

CH-8028 Zurich

E-mail: info@toxi.ch 


\section{Figure 1}

Evolution des intoxications non combinées aux ISRS ( $\mathrm{n}=197$ ).

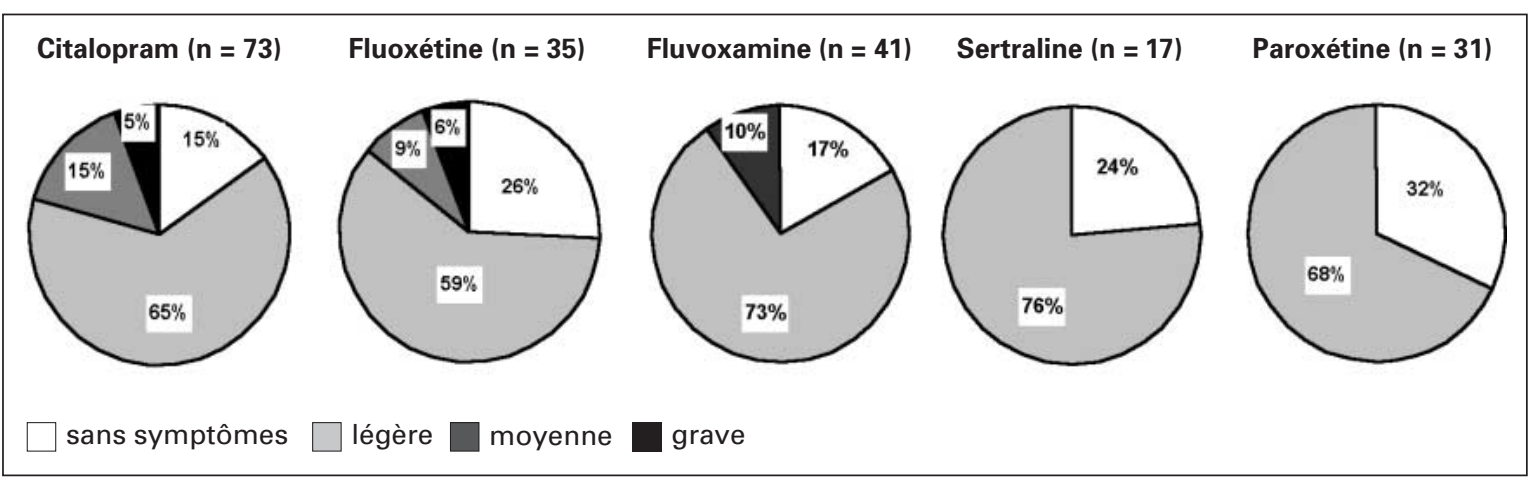

dangereux comme les produits fortement corrosifs ou les médicaments à haute toxicité. Parmi les produits de ménage, l'attention se porte actuellement sur les nouveaux nettoyants antibactériels. De premières expériences font penser que leurs additifs désinfectants peuvent provoquer des irritations supplémentaires des yeux et des muqueuses.

Chez l'adolescent (12 à 16 ans, 204 cas), ce sont l'abus et les intoxications intentionnelles qui prédominent. Dans le premier cas, on a vu des intoxications moyennes et graves avec l'alcool, le dextrométhorphane, les champignons hallucinogènes, la morphine, la cocaïne et le méthylphénidate (Ritaline ${ }^{\circledR}$ ). Sont apparus pour la première fois dans les statistiques du Tox cette année l'A2 (benzylpipérazine), le 2C-B (bromodiméthoxyphénéthylamine) et les pilules Thaï (méthamphétamine). Quand aux agents absorbés dans le cadre d'une conduite suicidaire, ils étaient très variés: les jeunes ont pris ce qui se trouvait dans la pharmacie familiale. Des intoxications graves et moyennes ont été signalées, par ordre diminuant, avec l'acide méfénamique, le paracétamol, les benzodiazépines, les bétabloquants, les inhibiteurs sélectifs du recaptage de la sérotonine et les antidépresseurs tricycliques.

Chez l'adulte, seuls 350 cas (12,1\%) sont restés sans symptômes. Les suites ont été bénignes dans 1642 cas (56,6\%), moyennes dans 650 (22,4\%), graves dans $242(8,4 \%)$ et mortelles dans 15 cas $(0,5 \%)$. 9 cas mortels ont résulté de l'ingestion intentionnelle de médicaments. Les drogues, le parathion et l'acide formique concentré sont cités chacun une fois. D'autres cas mortels se sont produits par un surdosage accidentel de méthotréxate, une défaillance hépatique induite par le naproxène, et un accident du travail avec l'acide fluorhydrique.

\section{Problèmes analysés spécialement en 2000}

Intoxications à la méthadone chez l'enfant en bas âge

Nous avons déjà signalé plus haut l'accident mortel d'un enfant ayant pris de la méthadone. Ce cas tragique nous a incité à rassembler tous les cas d'acci- dents avec la méthadone chez les jeunes enfants des dix dernières années (tab. 2). Nous avons trouvé 34 cas médicalement bien documentés. On constate que les jeunes enfants trouvent la méthadone en général dans des flacons non rangés que les parents ont obtenu, dans le cadre du traitement d'une toxicomanie aux opiacés, d'un médecin ou d'un pharmacien. 15 jeunes enfants ont ainsi été victimes d'une intoxication moyenne, grave ou même fatale. Les symptômes les plus fréquents étaient somnolence ou coma, miosis et insuffisance respiratoire. On a aussi observé des vomissements, une rétention urinaire, une défaillance circulatoire et, parmi les complications, une pneumonie par aspiration et un dommage cérébral hypoxique irréversible. Deux enfants sont morts à la suite d'une ingestion accidentelle de méthadone.

L'ingestion de méthadone chez le jeune enfant est certes un événement rare, mais non exempt de danger, comme le montrent nos cas. Une dose de $10 \mathrm{mg}$ peut suffire pour une intoxication mortelle. Les enfants touchés avalent en général la solution prête à être bue et souvent édulcorée au sirop que leurs parents ont reçu. Il ne s'agit pas ici de mettre en question le traitement de substitution à la méthadone, qui est bien établi. Les médecins et les pharmaciens qui donnent la méthadone pour l'utilisation à domicile à des parents sont par contre invités à procéder à toutes les mesures possibles pour empêcher une ingestion accidentelle par un enfant. Là où il n'est pas possible d'éviter l'emploi de la méthadone à domicile, les parents devraient être explicitement informés du danger que cela comporte pour leur fils ou leur fille, et le produit ne devrait être délivré que dans un flacon de sécurité.

Inhibiteurs sélectifs du recaptage de la sérotonine 333 intoxications chez l'adulte par un antidépresseur figurent dans la statistique du Tox pour 2000. A côté des antidépresseurs tricycliques, dont la toxicité qualitative et quantitative est bien connue, on trouve aussi de plus en plus des substances plus récentes, c'est-à-dire les inhibiteurs sélectifs du recaptage de la sérotonine (ISRS).

Dans le but d'une meilleure évaluation du risque, nous avons analysé 197 mono-intoxications aux 
Tableau 1

Groupes d'agents responsables et gravité des intoxications chez l'adulte et l'enfant.

\begin{tabular}{|c|c|c|c|c|c|c|c|c|c|c|c|c|}
\hline \multirow[b]{2}{*}{ Agents/Gravité } & \multicolumn{5}{|c|}{ Adultes } & \multicolumn{5}{|c|}{ Enfants } & \multicolumn{2}{|l|}{ Total } \\
\hline & 0 & $\mathrm{~L}$ & M & G & $\mathrm{F}$ & 0 & $\mathrm{~L}$ & M & G & $\mathrm{F}$ & & \\
\hline Médicaments & 264 & 1126 & 424 & 161 & 11 & 263 & 263 & 58 & 15 & 1 & 2586 & $(65,9 \%$ \\
\hline Produits domestiques & 28 & 123 & 20 & 4 & - & 83 & 89 & 6 & 6 & - & 359 & $(9,1 \%$ \\
\hline Produits techniques et industriels & 20 & 169 & 46 & 10 & 2 & 24 & 26 & 3 & 1 & - & 301 & $(7,7 \%$ \\
\hline Produits d'agrément, drogues et alcool & 9 & 72 & 83 & 50 & 1 & 16 & 19 & 4 & 2 & - & 256 & $(6,6 \%$ \\
\hline Plantes & 8 & 27 & 17 & - & - & 25 & 15 & 3 & - & - & 95 & $(2,4 \%$ \\
\hline Champignons & 3 & 17 & 26 & 4 & - & 4 & 1 & 3 & 2 & - & 60 & $(1,5 \%$ \\
\hline Produits d'agriculture et d'horticulture & 6 & 19 & 9 & 6 & 1 & 21 & 6 & - & - & - & 68 & $(1,7 \%$ \\
\hline Articles de toilette et produits cosmétiques & 2 & 19 & 3 & - & - & 17 & 10 & - & - & - & 51 & $(1,3 \%$ \\
\hline Animaux venimeux & 1 & 15 & 4 & 4 & - & 2 & 7 & 3 & - & - & 36 & $(0,9 \%$ \\
\hline Aliments et boissons & 2 & 4 & 3 & 1 & - & 1 & 6 & - & - & - & 17 & $(0,4 \%$ \\
\hline Produits à usage vétérinaire & 3 & 1 & 4 & - & - & - & - & - & - & - & 8 & $(0,2 \%$ \\
\hline Autres agents ou non classifiables & 4 & 50 & 11 & 2 & - & 1 & 21 & - & - & - & 89 & $(2,3 \%$ \\
\hline Total & 350 & 1642 & 650 & 242 & 15 & 457 & 463 & 80 & 26 & 1 & 3926 & $(100 \%)$ \\
\hline
\end{tabular}

Gravité de l'évolution: 0 = sans symptômes ou sans précisions, $\mathrm{L}$ = intoxications légères, $\mathrm{M}$ = intoxications moyennes,

$\mathrm{G}=$ intoxications graves, $\mathrm{F}=$ intoxications fatales.

\section{Tableau 2}

Evolution des accidents à la méthadone chez l'enfant en bas âge (1991-2000; $\mathrm{n}=34$ ).

\begin{tabular}{llll}
\hline Evolution & Nombre de cas & Symptômes principaux & Remarques \\
\hline sans symptômes & 6 & - & $\begin{array}{l}5 \text { des } 6 \text { enfants ont été rapidement décontaminés; } \\
\text { un enfant a reçu une dose prophylactique } \\
\text { de Narcan }{ }^{\circledR}\end{array}$ \\
\hline légère & 13 & $\begin{array}{l}\text { somnolence, vomissements, miosis, } \\
\text { troubles de la marche }\end{array}$ & $\begin{array}{l}7 \text { des } 13 \text { enfants ont été rapidement décontaminés; } \\
6 \text { enfants ont reçu par prudence du Narcan }{ }^{\circledR}\end{array}$ \\
\hline moyenne & 4 & $\begin{array}{l}\text { coma (GCS 8-9), dépression respiratoire } \\
\text { modérée }\end{array}$ & 3 enfants ont reçu du Narcan ${ }^{\circledR}$ \\
\hline grave & 9 & $\begin{array}{l}\text { coma (GCS } \leq 7), \text { dépression respiratoire, } \\
\text { oedème pulmonaire, dommage cérébral } \\
\text { hypoxique }\end{array}$ & $\begin{array}{l}8 \text { enfants ont reçu du Narcann }{ }^{\circledR}, \\
2 \text { onté intubés et ventilés }\end{array}$ \\
\hline fatale & 2 & défaillance respiratoire et circulatoire & $\begin{array}{l}\text { les deux enfants ont été victimes d'une défaillance } \\
\text { respiratoire et circulatoire avant l'arrivée } \\
\text { du médecin }\end{array}$ \\
\hline
\end{tabular}

ISRS bien documentées (cas signalés de 1984 à 2000). 18 cas étaient de gravité moyenne et 6 cas étaient graves, avec surtout des convulsions et des troubles de l'ECG. Chez les adultes, on a vu des symptômes graves avec des doses en excès de $280 \mathrm{mg}$ de citalopram, $60 \mathrm{mg}$ de fluoxétine et 1,5 g de fluvoxamine. La sertraline et la paroxétine n'ont pas provoqué de convulsions ou de troubles de l'ECG et n'ont occasionné que des intoxications bénignes. La figure 1 montre la part différente de cas sérieux pour ces cinq ISRS, soit 20\% pour le citalopram, 15\% pour la fluoxétine et $10 \%$ pour la fluvoxamine, envers $0 \%$ pour la sertraline et la paroxétine.

\section{Erreurs dans l'emploi des médicaments}

Le Tox reçoit régulièrement des appels de professionnels de la santé et du public lorsqu'une erreur a été constatée dans l'emploi d'un médicament. Les cas correspondants des années 1998 à 2000 ont été étudiés dans le cadre d'une étude-pilote. Seuls les cas où l'appelant a déclaré qu'il s'agissait d'une erreur ont été inclus dans l'analyse. Le nombre total de ces cas est de 1035. Il s'agissait le plus souvent d'un surdosage par méprise ou d'une confusion (du médicament ou du patient), portant souvent sur des produits psychoactifs ou des analgésiques. L'auteur de l'erreur était une personne sans connaissances médicales 
dans $68 \%$ des cas, et une personne du domaine médical (médecin, pharmacien, soignant) dans le restant des cas. Dans un tiers environ des cas le médicament était administré par la victime elle-même, dans deux tiers des cas par une autre personne. $46 \%$ des personnes touchées étaient des enfants ( $\leq 16$ ans). Les appels pour des erreurs dans l'emploi d'un médicament étaient deux fois aussi fréquents entre 19 et $23 \mathrm{~h}$ que pendant la journée. Une explication pourrait être que des personnes compétentes sont plus difficiles à atteindre le soir, tandis que le Tox est disponible jour et nuit. Les données rassemblées montrent en tout cas que le Tox est appelé à répondre non seulement en cas d'intoxication, mais aussi lors de médications erronées.

\section{Conclusions}

Des données toxicologiques chez l'homme sur les intoxications et les fausses applications ne peuvent, pour des raisons évidentes, être obtenues par des études expérimentales. Le système de communications spontanées, de son côté, ne permet pas non plus au Tox de produire des données fiables quant à la fréquence absolue des intoxications et fausses applica- tions. Les appels et les communications sont par contre des signaux pour les problèmes toxicologiques actuels et nouveaux dans la population, et peuvent servir de système de reconnaissance précoce en toxicovigilance. Cela vaut en particulier pour l'abus de drogues nouvelles, pour la tolérabilité de médicaments nouveaux et pour les risques propres aux produits nouveaux pour le ménage, l'artisanat, l'industrie et l'agriculture. Les intoxications à la méthadone chez l'enfant montrent une fois de plus que le système de communications spontanées procure aussi des notions actuelles et à implications préventives pour des substances établies. La valeur des observations médicales sur l'évolution des intoxications chez l'homme, enfin, n'est plus à prouver. Ces rapports médicaux permettent au Tox de développer de manière continue la banque des intoxications qui constitue la base pour l'évaluation des risques pour l'homme des produits et substances nouvelles et anciennes et la base aussi pour des analyses de toxicité comparée comme dans le cas des différents inhibiteurs du recaptage de la sérotonine. Le Tox dit merci à tous les médecins et pharmaciens hospitaliers et praticiens qui par leur coopération permettent en Suisse une amélioration continuelle de la sécurité des médicaments et des produits chimiques en général. 